\title{
BOUNDED P.S.H. FUNCTIONS AND PSEUDOCONVEXITY IN KÄHLER MANIFOLD
}

\author{
TAKEO OHSAWA AND NESSIM SIBONY
}

\begin{abstract}
It is proved that the $C^{2}$-smoothly bounded pseudoconvex domains in $\mathbb{P}^{n}$ admit bounded plurisubharmonic exhaustion functions. Further arguments are given concerning the question of existence of strictly plurisubharmonic functions on neighbourhoods of real hypersurfaces in $\mathbb{P}^{n}$.
\end{abstract}

Let $\Omega \Subset M$ be a pseudoconvex domain in a Kähler manifold $M$. When $M$ is $\mathbb{P}^{k}$, Takeuchi [T], showed that the function - $\log \delta_{\Omega}$ is strictly plurisubharmonic (p.s.h.) in $\Omega$. Here $\delta_{\Omega}$ denotes the distance to the boundary for the standard Kähler metric on $\mathbb{P}^{k}$.

The result was extended by Elencwajg [E] to the case where $M$ is Kähler with strictly positive holomorphic bisectional curvature. See also Suzuki [Su] and Green-Wu [G.W].

Based on their result we show that if $\Omega \Subset M$ is pseudoconvex with $\mathcal{C}^{2}$ boundary, then there is a bounded strictly p.s.h. function on $\Omega$. When $M=\mathbb{C}^{k}$ the question was solved by Diedrich-Fornaess [D.F]. For a survey in this case see $[\mathrm{S}]$.

We give an example of a compact Kähler manifold $M$, containing a Stein domain $\Omega \Subset M$, with smooth boundary, however given any neighborhood $U$ of $\partial \Omega$, there is no nonconstant bounded p.s.h. function on $U \cap \Omega$.

We show next that the existence of a strictly p.s.h. function near $\partial \Omega$ is equivalent to the nonexistence of a positive current $T$ of bidimension $(1,1)$ supported on $\partial \Omega$ and satisfying the equation $\partial \bar{\partial} T=0$. This result is inspired by a duality argument due to Sullivan $[\mathrm{Su}]$.

\section{$\S 1$. Plurisubharmonic exhaustion function on smoothly bounded domains}

Let $(M, \omega)$ be a Kähler manifold. Let $\Omega \Subset M$ be a pseudoconvex domain with smooth boundary. We consider first the question of existence of a strictly plurisubharmonic bounded exhaustion function for $\Omega$.

Received September 27, 1996. 
TheOREM 1.1. Let $\Omega \Subset M$ be a pseudoconvex domain with $\mathcal{C}^{2}$ boundary in a complete Kähler manifold $M$. Assume the holomorphic bisectional curvature of $M$ is strictly positive. Let $r(z)=-\operatorname{dist}(\mathrm{z}, \partial \Omega)=: \delta(\mathrm{z})$ where $\delta$ is computed with respect to the Kähler metric. Then there exists $\varepsilon>0$ such that $\varphi=-(-r)^{\varepsilon}$ is strictly plurisubharmonic in $\Omega$. More precisely there is a constant $c_{\varepsilon}$ such that

$$
i \partial \bar{\partial} \varphi \geq c_{\varepsilon}|\varphi| \omega
$$

Proof. Under the above assumption on the curvature, Takeuchi $[\mathrm{T}]$ for the projective space, Elencwajg [E], in general, proved that $-\log \delta$ is strictly plurisubharmonic. More precisely there is a constant $C$ depending on the lower bound for the curvature, such that

$$
i \partial \bar{\partial}(-\log \delta) \geq C \omega
$$

So if $r=-\delta$ we get

$$
-r i \partial \bar{\partial} r+i \partial r \wedge \bar{\partial} r \geq C r^{2} \omega
$$

We can choose local coordinates near $p \in \partial \Omega$, such that $x_{2 n}=r, e_{i}(r)=0$, $i=1, \ldots, n-1$, where $\left(e_{i}\right)$ is an orthonormal basis for the complex tangent space to $\partial \Omega$ near $p$. Let $\left(a_{i j}\right)$ denote the hermitian form corresponding to $i \partial \bar{\partial} r$. Inequality (1) gives in coordinates

$$
-r \sum_{i, j=1}^{n} a_{i j} v_{i} \bar{v}_{j}+|\partial r|^{2}\left|v_{n}\right|^{2} \geq C r^{2} \sum_{j=1}^{n}\left|v_{j}\right|^{2} .
$$

If $v_{n}=0$ we obtain the estimate

$$
\sum_{i, j=1}^{n-1} a_{i j} v_{i} \bar{v}_{j} \geq C|r| \sum_{j=1}^{n-1}\left|v_{j}\right|^{2}
$$

Expanding (2) we get

$$
\begin{aligned}
& -r \sum_{i, j=1}^{n-1} a_{i j} v_{i} \bar{v}_{j}+2 \operatorname{Re}(-r) \sum_{k=1}^{n-1} a_{n k} v_{n} \bar{v}_{k}-r a_{n n}\left|v_{n}\right|^{2}+|\partial r|^{2}\left|v_{n}\right|^{2} \\
\geq & C r^{2} \sum_{j=1}^{n}\left|v_{j}\right|^{2} .
\end{aligned}
$$


Replacing $v$, by $v_{j} /(-r)$ for $j \leq n-1$ we obtain

$$
\begin{aligned}
& \sum_{i, j=1}^{n-1} \frac{a_{i j}}{(-r)} v_{i} \bar{v}_{j}+2 \operatorname{Re} \sum_{k=1}^{n-1} a_{n k} v_{n} \bar{v}_{k}-r a_{n n}\left|v_{n}\right|^{2}+|\partial r|^{2}\left|v_{n}\right|^{2} \\
\geq & C \sum_{j=1}^{n-1}\left|v_{j}\right|^{2} .
\end{aligned}
$$

We write the left hand side of this inequality as

$$
Q(z, v)+|\partial r|^{2}\left|v_{n}\right|^{2}
$$

Let $\tilde{Q}(\zeta, v):=\liminf _{\substack{z \rightarrow \zeta \\ z \in \Omega}} Q(z, v)=\lim _{s \rightarrow 0} \inf _{\substack{|z-\zeta|<s \\ z \in \Omega}} Q(z, v)$. From (3) we obtain

$$
\tilde{Q}(\zeta, v)+|\partial r|^{2}(\zeta)\left|v_{n}\right|^{2} \geq C \sum_{j=1}^{n-1}\left|v_{j}\right|^{2} .
$$

Observe that $\tilde{Q}\left(p,\left(0, v_{n}\right)\right) \geq 0$. So by the lower semicontinuity of $\tilde{Q}$, for $c$ small enough

$$
\tilde{Q}(\zeta, v)+|\partial r|^{2}(\zeta)\left|v_{n}\right|^{2}>c\left|v_{n}\right|^{2}
$$

in a neighborhood of $p$. Inequality (5) remains valid in a neighborhood of $v^{\prime}=0$, i.e. for $\left|v^{\prime}\right| \leq \alpha$, on the sphere $|v|=1$, where $v=\left(v^{\prime}, v_{n}\right)$.

We get then that

$$
Q(z, v)+|\partial r|^{2}(z)\left|v_{n}\right|^{2} \geq \frac{c}{2}\left|v_{n}\right|^{2}
$$

for $\delta(z)<\beta,\left|v^{\prime}\right| \leq \alpha$. But, when $\left|v^{\prime}\right|>\alpha$ and $|v|=1$ we have $\left|v^{\prime}\right|^{2} \geq$ $\varepsilon_{0}\left|v_{n}\right|^{2}$, where $\varepsilon_{0}=\alpha^{2}\left(1-\alpha^{2}\right)^{-1}$.

So using (4) we get that

$$
Q(z, v)+|\partial r|^{2}\left|v_{n}\right|^{2} \geq \varepsilon^{\prime}\left|v_{n}\right|^{2} \quad \text { for some } \varepsilon^{\prime}>0
$$

and for $\delta(z)<\beta$. This implies

$$
Q(z, v)+|\partial r|^{2}\left|v_{n}\right|^{2} \geq \frac{\varepsilon^{\prime}}{2}\left|v_{n}\right|^{2}+\frac{c}{2} \sum_{j=1}^{n-1}\left|v_{j}\right|^{2} .
$$

Rescaling this we obtain

$$
-r \sum_{i, j=1}^{n} a_{i j} v_{i} \bar{v}_{j}+|\partial r|^{2}\left|v_{n}\right|^{2} \geq \frac{\varepsilon}{2}\left|v_{n}\right|^{2}+\frac{c}{2} \sum_{j=1}^{n-1}\left|v_{j}\right|^{2}
$$


which can be read as

$$
-i \partial \bar{\partial}(-r)^{\varepsilon}=i \varepsilon(-r)^{\varepsilon}\left(\frac{\partial \bar{\partial} r}{-r}+(1-\varepsilon) \frac{\partial r \wedge \bar{\partial} r}{r^{2}}\right) \geq \frac{c}{2} \varepsilon|r|^{\varepsilon} \omega .
$$

The condition of positivity of holomorphic sectional curvature in order to construct a strictly p.s.h. bounded exhaustion function seems quite sharp. Indeed we have the following result.

THEOREM 1.2. There is a compact Kähler surface $M$ which has the following property. There is $\Omega \Subset M$ a Stein domain with real analytic boundary with $\partial \Omega$ Levi-flat, such that for every neighborhood $U$ of $\partial \Omega$ there is no nonconstant bounded p.s.h. function on $U \cap \Omega$.

Proof. $M$ will be given as the quotient of $\mathbb{C} \times \mathbb{P}^{1}$ under a $\mathbb{Z}^{2}$ action. For $(a, b) \in \mathbb{Z}^{2}$ let $f_{a, b}(z, \omega)=(z+a+b \omega, w+a+\alpha b)$ where $\omega \in \mathbb{C} \operatorname{Im} \omega>0$, and $\alpha \in \mathbb{R} \backslash \mathbb{Q}$ are fixed. Here $w$ denotes an inhomogeneous coordinate on $\mathbb{P}^{1}$. It is clear that $M$ is a compact surface. $M$ is also a $\mathbb{P}^{1}$-bundle on the torus $A=\mathbb{C} / \mathbb{Z}^{2}$. Hence $M$ is Kähler. We can also observe that $M$ is homogeneous in the sense that the tangent bundle is generated by global holomorphic vector fields.

We observe that $M$ is foliated by complex leaves. Let $\pi: \mathbb{C} \times \mathbb{P}^{1} \rightarrow M$ be the canonical projection. For $w_{0}$ fixed $\pi$ is injective on $\mathbb{C} \times w_{0}$, because $\alpha \notin \mathbb{Q}$. We also have that $\pi\left(\mathbb{C} \times w_{0}\right)=\pi\left(\mathbb{C} \times w_{1}\right)$ iff $w_{1}=w_{0}+a_{0}+\alpha b_{0}$. It follows that for any $y_{0} \in \mathbb{R} L_{y_{0}}:=\pi\left(\mathbb{C} \times y_{0}\right) \supset \pi\left(\mathbb{C} \times \operatorname{Im} w=y_{0}\right) \cup A_{1}$, where $A_{1}$ denote the torus $\pi(\mathbb{C} \times \infty)$. It is then clear that the closure of each leaf (except for $A_{1}$ ) contains a Levi-flat hypersurface which is a real analytic three dimensional torus. Define $\Omega:=\pi(\operatorname{Im} w>0)$. Then $\partial \Omega$ is real analytic and Levi-flat. Let $U$ be a neighborhood of $\partial \Omega$. Assume $\varphi U \cap \Omega \rightarrow\left[-c_{1}, 0[\right.$ is p.s.h. For $0<y_{0}<\varepsilon_{0}, \varepsilon_{0} \ll 1 L_{y_{0}}$ is contained in $U$. Since $\varphi$ is bounded above it is constant on $L_{y_{0}}=\pi\left(\operatorname{Im} w=y_{0}\right)$. Fix $p \in \overline{L_{y_{0}}} \cap U$. Choose $\varepsilon>0$ small enough so that $B(p, \varepsilon) \subset U$. Let $c:=\max \varphi_{\bar{B}(p, \varepsilon) \cap \overline{L_{y_{0}}}}$. The closed set $(\varphi \geq c)$ is invariant under the foliation. So $\varphi=c$ on $\overline{L_{y_{0}}}$. As a consequence $\varphi$ is just a function of $y$, i.e. $\varphi=h(y), h$ defined for $0<y<\varepsilon_{0}$. The plurisubharmonicity of $\varphi$ implies that $w \rightarrow h(y)$ is subharmonic so $h$ is convex with respect to $y$. The function is defined for $y>0$ bounded hence constant. 
The domain $\Omega$ is Stein. Indeed the function $\pi(z, w) \rightarrow \sup (-\log |y|,|y|)$ is a p.s.h. exhaustion function on $\Omega$. Since $M$ is homogeneous and $\Omega$ does not contain a relatively compact leaf, it follows form a theorem of Hirshowitz $[\mathrm{H}]$ that $\Omega$ is Stein.

\section{§2. Strictly p.s.h. functions near $\partial \Omega$}

Let $\Omega \Subset M$ be a pseudoconvex domain with $\mathcal{C}^{2}$ boundary in the complex manifold $M$. We are interested in the existence of a strictly p.s.h. function in a neighborhood of $\partial \Omega$. The examples of in the previous paragraph show that this is not always the case, even when $\Omega$ is Stein. Our result is inspired by the duality principle from Sullivan $[\mathrm{Su}]$. Recall that currents of bidimension $(1,1)$ act on forms of bidegree $(1,1)$. Let $X$ be a closed subset of $M$. Assume $x \rightarrow \alpha_{x}$ is a continuous map on $X$ with values in complex linear maps, $\alpha_{x}$ is allowed to be zero on some subset of $X$.

Definition 2.1. A positive current $T$, of bidimension $(1,1)$, is directed by $\operatorname{ker} \alpha_{X}$ iff $T \wedge i \alpha_{x} \wedge \bar{\alpha}_{x}=0$ on $X$.

The positivity of $T$ implies that $T \wedge i \alpha_{x} \wedge \bar{\alpha}_{x}$ is a positive measure, so we are asking that this measure vanishes on $X$.

If we assume that $T$ is supported on $X$, this is equivalent to the fact that $T$ belongs to the closure of the convex cone generated by the currents $\varepsilon_{x}\left(i \xi_{n} \otimes \bar{\xi}_{n}\right)$ where $\alpha_{x}\left(\xi_{x}\right)=0$ and $\varepsilon_{x}$ denote the Dirac mass at $x$. We will consider $M$ as a hermitian manifold, which allows one to give a norm $T$ to positive currents, i.e. $\|T\|=\langle T, \omega\rangle$ where $\omega$ is a fixed strictly positive $(1,1)$-form.

Theorem 2.2. Let $\Omega \Subset M$ be a pseudoconvex domain with $\mathcal{C}^{2}$ boundary. The following are equivalent.

i) There is a smooth strictly plurisubharmonic function near $\partial \Omega$.

ii) There is no, nontrivial, positive current $T$, of bidimension $(1,1)$ supported on $\partial \Omega$ and directed by the complex tangent spaces to $\partial \Omega$, satisfying the equation $i \partial \bar{\partial} T=0$.

Proof. Assume i). Let $T$ be positive $(1,1)$ and supported on $\partial \Omega$. Let $\varphi$ be a strictly p.s.h. function near $\partial \Omega$. Then if $T$ is non-zero,

$$
0<\langle T, i \partial \bar{\partial} \varphi\rangle=\langle i \partial \bar{\partial} T, \varphi\rangle=0
$$


a contradiction. We now show ii) implies i). Let $\rho$ be a $\mathcal{C}^{2}$ defining function for $\partial \Omega$. Define $C=\{T \mid T \geq 0,(1,1),\|T\|=1, T$ directed by ker $\partial \rho$. $\}$ The set is convex and compact for the topology of currents. If i) does not hold then $C \cap\{i \partial \bar{\partial} u\}^{\perp}=\emptyset$, here $\{i \partial \bar{\partial} u\}^{\perp}$ denote the orthogonal space of $\{i \partial \bar{\partial} u\}$ when $u$ is a test function on $M$, i.e. a smooth function on $M$, so the space is closed. Using Hahn-Banach and reflexivity for the space of test functions we get the existence of $\psi \in \operatorname{closure}\{i \partial \bar{\partial} u\}$ such that $\langle T, \psi\rangle>0$ for every $T$ in $C$. Since $C$ is compact we can assume that $\psi=i \partial \bar{\partial} u$.

If $T=\varepsilon_{x} i \xi \otimes \bar{\xi}$ we get that $\langle i \partial \bar{\partial} u(x) \xi \wedge \bar{\xi}\rangle>0$, for $x \in \partial \Omega$ and $\xi$ complex tangent.

Define $\varphi_{\lambda}=u+\frac{e^{\lambda \rho}-1}{\lambda}$. For $\lambda \gg 1$, the pseudoconvexity of $\partial \Omega$ implies that $\varphi_{\lambda}$ is strictly p.s.h. near $\partial \Omega$.

Without assuming $\partial \Omega$ smooth we get easily the following.

THEOREM 2.3. Let $\Omega \Subset M$. The following are equivalent.

i) There is a smooth strictly p.s.h. function near $\partial \Omega$.

ii) There is no, nontrivial, positive $(1,1)$ current $T$ supported on $\partial \Omega$ such that $i \partial \bar{\partial} T=0$.

It is of interest to localize the support of such pluriharmonic currents i.e. positive currents satisfying $i \partial \bar{\partial} T=0$. Assume $\Omega \Subset M$. We define $J \subset \partial \Omega$ as the set of $x \in \partial \Omega$ such that there exists a Stein neighborhood $U \ni x$, and a p.s.h. function $\varphi_{x}$ defined near $\bar{U}$ with $\varphi_{x}(x)>0$ and $\sup _{\partial \Omega \cap \partial U} \varphi_{x}<0$.

Shrinking $U$ we can assume the existence of a strictly p.s.h. function $\rho$, on neighborhood of $\bar{U}, \varphi_{x}+\varepsilon \rho$, with $0<\varepsilon \ll 1$, will be strictly p.s.h. near $x$ and will have the same properties as $\varphi_{x}$ otherwise. Composing with a convex increasing function, we can assume $\varphi_{x}$ vanishes identically in a neighborhood of $\partial \Omega \cap \partial U$, with respect to $\partial \Omega$. We call $J$ the weak Jensen boundary of $\partial \Omega$. Clearly $J$ is open and contains the points of strict pseudoconvexity of $\partial \Omega$, when $\partial \Omega$ is of class $\mathcal{C}^{2}$.

THEOREM 2.4. Assume $\Omega \Subset M$ is pseudoconvex with $\mathcal{C}^{2}$ boundary. Let $T$ be a pluriharmonic positive current directed by the complex tangent space to $\partial \Omega$. Then the support of $T$ is contained in the complement of $J$, the weak-Jensen boundary of $\partial \Omega$. 
Proof. Let $x \in J$. Choose $\varphi$ a p.s.h. function in $U$, strictly p.s.h. near $x$, vanishing on a neighborhood in $\partial \Omega$, of $\partial U \cap \partial \Omega$. If $T$ is a positive $(1,1)$ current directed by the complex tangent space to $\partial \Omega$ we get

$$
\langle T, i \partial \bar{\partial} \varphi\rangle=\langle i \partial \bar{\partial} T, \varphi\rangle \text {. }
$$

The integration by part is possible because we consider $T$ as a current on $\partial \Omega$, and $\varphi$ as a function with compact support on $U \cap \partial \Omega$.

It is of interest to consider the possibility of existence of positive closed currents with support on the boundary of a pseudoconvex domain $\Omega \Subset M$. This is possible for domains in a $\mathbb{P}^{1}$ bundle over a Riemann surface or in a complex torus. However in $\mathbb{P}^{2}$, this is not possible.

ThEOREM 2.5. Let $\Sigma$ be a hypersurface of class $\mathcal{C}^{2}$ in $\mathbb{P}^{2}$. Then there is no positive $(1,1)$ closed current $T$ supported on $\Sigma$.

Proof. Let $\Omega_{1}, \Omega_{2}$ be the components of $\mathbb{P}^{2} \backslash \Sigma$. Let $\omega$ be the standard Kähler form in $\mathbb{P}^{2}$. Suppose there are 2-cycles $\sigma_{1} \subset \Omega_{1}, \sigma_{2} \subset \Omega_{2}$ such that $\left\langle\sigma_{j}, \omega\right\rangle=a_{j} \neq 0$. Since the second Betti number of $\mathbb{P}^{2}$ is 1 , by Poincaré duality $\sigma_{j} \sim a_{j} \omega$. But $\sigma_{1} \wedge \sigma_{2}=0$ and $a_{1} a_{2} \omega \wedge \omega \neq 0$ a contradiction. So we can assume that for every 2 cycle $\sigma$ in a neighborhood of $\bar{\Omega}_{1}$ we have $\langle\sigma, \omega\rangle=0$. We are using here that $\Omega_{1}$ is smoothly bounded. By De Rham Theorem there is a smooth form $\varphi$ such that $d \varphi=\omega$ in a neighborhood of $\Omega_{1}$. Let $T$ be a positive closed current of bidimension $(1,1)$ supported on $\Sigma$. Then if $T$ is nonzero

$$
0<\langle T, \omega\rangle=\langle T, d \varphi\rangle=\langle d T, \varphi\rangle=0
$$

So $T=0$.

Remark. Let $\Sigma$ be a real hypersurface in $\mathbb{P}^{k}$. We prove similarly that there is no non-zero positive closed current of bidimension $(1,1)$ supported on $\Sigma$. We get

$$
\left\langle T, \omega^{k-1}\right\rangle=\left\langle T, d\left(\varphi \wedge \omega^{k-2}\right)\right\rangle=0 .
$$

In particular there is no one dimensional complex curve on $\Sigma$.

Acknowledgements. The second author thanks Ngaiming Mok for pointing out that the manifold $M$ in Theorem 1.2 is not a manifold with nonnegative holomorphic bisectional curvature (see $[\mathrm{M}]$ ). 


\section{REFERENCES}

[D.F] Diederich, K., Fornaess, J. E., Pseudoconvex domains: Bounded strictly plurisubharmonic functions, Invent. Math., 39 (1977), 129-141.

[E] Elencwajg, G., Pseudoconvexité locale dans les variétés kähleriennes, Ann. Inst. Fourier, 25 (1975), 295-314.

[G.W] Greene, R. E. and Wu, H., On Kähler manifolds of positive bisectional curvature and a theorem of Hartogs, Abh. Math. Sem. Univ. Hamburg, 47 (1978), 171-185.

[H] Hirshowitz, A., Pseudoconvexité au dessus d'espaces plus ou moins homogenes, Invent. Math., 26 (1974), 303-322.

[M] Mok, N., The uniformization theorem for compact Kähler manifolds of monnegative holomorphic bisectional curvature, J. Differential Geom., 27 (1988), 179-214.

[S] Sibony, N., some aspects of weakly pseudoconvex domains in several complex variables and complex geometry, Proceedings of symposia in Pure Math., 62 part 1 (1991), 199-231.

[Suz] Suzuki, O., Pseudoconvex domains on a Kähler manifold with positive holomorphic bisectional curvature, Pubk. Res. Inst. Math. Sci., 12 (1976), 191-214.

[Su] Sullivan, D., Cycles for dynamical study of foliated manifolds and complex manifolds, Invent. Math., 36 (1976), 225-255.

[T] Takeuchi, A., Domains pseudoconvexes infinis et la metrique riemannienne daus un espace projectif, J. Math. Soc. Japan, 16 (1964), 159-181.

Takeo Ohsawa

Graduate School of Mathematics

Nagoya University

Chikusa-ku, Nagoya 464-8602

Japan

Nessim Sibony

Univ. Paris Sud

Mathématiques, Bât 425

91405 Orsay

France 